\title{
Passive Control and $\varepsilon$-Bound Estimation of Singularly Perturbed Systems with Nonlinear Nonlinearities
}

\author{
Linna Zhou and Chunyu Yang \\ School of Information and Electrical Engineering, China University of Mining and Technology, Xuzhou 221116, China \\ Correspondence should be addressed to Chunyu Yang; chunyuyang@yahoo.cn
}

Received 9 March 2013; Accepted 14 April 2013

Academic Editor: Zheng-Guang Wu

Copyright (C) 2013 L. Zhou and C. Yang. This is an open access article distributed under the Creative Commons Attribution License, which permits unrestricted use, distribution, and reproduction in any medium, provided the original work is properly cited.

\begin{abstract}
This paper considers the problems of passivity analysis and synthesis of singularly perturbed systems with nonlinear uncertainties. By a novel storage function depending on the singular perturbation parameter $\varepsilon$, a new method is proposed to estimate the $\varepsilon$-bound, such that the system is passive when the singular perturbation parameter is lower than the $\varepsilon$-bound. Furthermore, a controller design method is proposed to achieve a predefined $\varepsilon$-bound. The proposed results are shown to be less conservative than the existing ones because the adopted storage function is more general. Finally, an RLC circuit is presented to illustrate the advantages and effectiveness of the proposed methods.
\end{abstract}

\section{Introduction}

Singularly perturbed systems (SPSs), with a small singular perturbation parameter $\varepsilon$ determining the degree of separation between the "slow" and "fast" modes of the systems, have attracted much attention due to their widespread applications in chemical process, robot, aerospace engineering, power systems, and so forth $[1,2]$. A key problem for investigations of SPSs is to estimate the upper bound $\bar{\varepsilon}$ of the singular perturbation parameter $\varepsilon$ such that the system preserves its stability or other desired performance for all $\varepsilon \in(0, \bar{\varepsilon}]$, which is referred to as the $\varepsilon$-bound problem [3-7]. The $\varepsilon$-bound problem on stability of SPSs has been widely considered and many frequency- and time-domain methods have been proposed [3-5]. $H_{\infty}$ performance of SPSs was considered in $[6,7]$, and some methods for evaluating the upper bound $\bar{\varepsilon}$ to meet a prescribed $H_{\infty}$ performance requirement were given. However, the $\varepsilon$-bound problem on passivity of SPSs is still open.

Passivity, which was introduced by Willems $[8,9]$, relates nicely to stability of systems. On one hand, storage functions induced by passivity are usually related with system energy and thus provide natural candidates for the Lyapunov functions; on the other hand, passivity is expected to be preserved under feedback interconnection, which provides a useful tool for stability analysis of feedback systems [10]. In addition, the passivity theory which is intimately related to circuit analysis methods has played an important role in robust and nonlinear stabilization problems $[11,12]$. It has been shown that passivity provides a suitable design approach in power systems, neural networks, signal processing, chaos control, and synchronization [11-16].

Recently, the problem of passivity analysis and synthesis of SPSs has attracted more attention. Applying the routine methods for normal systems to SPSs usually leads to ill-conditioned numerical problems [1]. The conventional approaches to avoiding the ill-conditioned problem are based on decomposing the original SPSs into fast and slow subsystems. In [17], passive control of T-S fuzzy SPSs was studied. It was shown that the passivity of SPSs is equivalent to the passivity of both the boundary system and the slow subsystem. The proposed method is based on the decomposition of the original systems, which leads to difficulties for computing the $\varepsilon$-bound for the SPSs to preserve passivity. An alternative approach to alleviating the ill-conditioned problem is based on the so-called descriptor system method and independent of the system decomposition [18-20]. Passivity analysis and passivity-based controller design were studied for uncertain singularly perturbed Markovian jump systems with timevarying delay in [18]; but it did not considered the $\mathcal{E}$ bound estimation problem. In [19], sufficient conditions in 
terms of linear matrix inequalities (LMIs) were derived, and the estimation of $\varepsilon$-bound can be obtained by solving a generalized eigenvalue problem (GEVP). Following the line of [19], passivity-based integral sliding mode control was studied for uncertain singularly perturbed systems in [20]. All in all, passive control of SPSs with the consideration of $\varepsilon$-bound estimation is still an open problem.

This paper considers passive control and $\varepsilon$-bound estimation of singularly perturbed systems with nonlinear uncertainties. A storage function which gives full consideration of the singular perturbation structure is first constructed, by which a sufficient condition for the system to be passive for any $\varepsilon \in(0, \bar{\varepsilon}]$ is proposed, where $\bar{\varepsilon}$ is an upper bound of $\varepsilon$. Then, based on the proposed sufficient condition, a bisectional search algorithm is formulated to compute the best estimate of $\varepsilon$-bound. Furthermore, a controller design method is proposed to achieve a predefined $\varepsilon$-bound. Finally, an RLC circuit is presented to demonstrate the proposed results. The main contributions of the paper are as follows: (1) the proposed method implicitly employs the singular perturbation structure of the SPSs rather than depends on decomposing the original systems into reduced-order subsystems, which provides convenience for solving the $\varepsilon$-bound problem; (2) the adopted storage function is more general than the existing one, which leads to a less conservative method for passivity analysis of SPSs and provides a tighter estimate of the $\varepsilon$-bound; (3) a constructive algorithm for computing the best estimate of the $\varepsilon$-bound is proposed; (4) a predefined $\varepsilon$-bound is one of the controller design objectives.

\section{Preliminaries}

This paper will consider the following singularly perturbed system:

$$
\begin{gathered}
E(\varepsilon) \dot{x}(t)=A x(t)+B_{f} f(t, x)+B_{u} u(t)+B_{\omega} \omega(t), \\
z(t)=C x(t)+D_{g} g(t, x)+D_{\omega} \omega(t),
\end{gathered}
$$

where $x(t)=\left[\begin{array}{ll}x_{1}^{T} & x_{2}^{T}\end{array}\right]^{T} \in R^{n}$ denotes the system state, $x_{1} \in$ $R^{n_{1}}$ and $x_{2} \in R^{n_{2}},\left(n_{1}+n_{2}=n\right)$ represent the slow modes and the fast modes of the state, respectively, $u(t) \in R^{l}$ is the control input, $z(t) \in R^{p}$ is the controlled output, and $\omega(t) \in L_{2}^{q}[0, \infty)$ is the external disturbance. And $E(\varepsilon)=\left[\begin{array}{cc}I_{n_{1}} & 0_{n_{1} \times n_{2}} \\ 0_{n_{2} \times n_{1}} & \varepsilon I_{n_{2}}\end{array}\right] \in R^{n \times n}$, $A \in R^{n \times n}, B_{f} \in R^{n \times r}, B_{u} \in R^{n \times l}, B_{\omega} \in R^{n \times q}, C \in R^{m \times n}$, $D_{g} \in R^{n \times s}$, and $D_{\omega} \in R^{m \times q}$ are known constant matrices. $f(t, x) \in R^{r}$ and $g(t, x) \in R^{s}$ are vector-valued time-varying nonlinear uncertainties, which are bounded by

$$
\begin{gathered}
\|f(t, x)\| \leq \alpha\|M x(t)\|, \\
\|g(t, x)\| \leq \beta\|N x(t)\|,
\end{gathered}
$$

where $\alpha>0, \beta>0$ are given constants and $M, N$ known constant matrices of approximate dimensions.

Definition 1 (see $[13,21]$ ). System (1) is said to be passive if

$$
\int_{0}^{T} \omega^{T}(t) z(t) d t \geq 0, \quad \forall T>0
$$

holds for all trajectories with zero initial condition $x(0)=0$. It is said to have dissipation $\eta$ if

$$
\int_{0}^{T} \omega^{T}(t) z(t)-\eta \omega^{T}(t) \omega(t) d t \geq 0, \quad \forall T>0
$$

holds for all trajectories with zero initial condition $x(0)=0$.

The largest dissipation of the system, that is, the largest value of $\eta$ such that (5) holds, is usually called its dissipativity $[13,21]$. It is known that a system with larger dissipativity is able to tolerate larger uncertainties and disturbances [13]. When $\eta=0$, it is the case of passivity that was discussed in [19]. In this paper, we will consider the more general case $\eta \geq 0$.

The following lemmas will be used in the sequel.

Lemma 2 (see [7]). Given $\bar{\varepsilon}>0$ and symmetric matrices $S_{1}, S_{2}$, and $S_{3}$, if

$$
\begin{gathered}
S_{1} \geq 0, \\
S_{1}+\bar{\varepsilon} S_{2}>0, \\
S_{1}+\bar{\varepsilon} S_{2}+\bar{\varepsilon}^{2} S_{3}>0,
\end{gathered}
$$

then, it holds that

$$
S_{1}+\varepsilon S_{2}+\varepsilon^{2} S_{3}>0, \quad \forall \varepsilon \in(0, \bar{\varepsilon}] .
$$

Lemma 3 (see [7]). If there exist matrices $Z_{i}(i=1,2, \ldots, 5)$ of appropriate dimensions with $Z_{i}=Z_{i}^{T}(i=1,2,3,4)$ satisfying the following LMIs:

$$
\begin{gathered}
Z_{1}>0 \\
{\left[\begin{array}{cc}
Z_{1}+\bar{\varepsilon} Z_{3} & \bar{\varepsilon} Z_{5}^{T} \\
\bar{\varepsilon} Z_{5} & \bar{\varepsilon} Z_{2}
\end{array}\right]>0} \\
{\left[\begin{array}{cc}
Z_{1}+\bar{\varepsilon} Z_{3} & \bar{\varepsilon} Z_{5}^{T} \\
\bar{\varepsilon} Z_{5} & \bar{\varepsilon} Z_{2}+\bar{\varepsilon}^{2} Z_{4}
\end{array}\right]>0}
\end{gathered}
$$

then, it holds that

$$
E(\varepsilon) Z(\varepsilon)=(E(\varepsilon) Z(\varepsilon))^{T}=Z^{T}(\varepsilon) E(\varepsilon)>0, \quad \forall \varepsilon \in(0, \bar{\varepsilon}],
$$

where

$$
Z(\varepsilon)=\left[\begin{array}{cc}
Z_{1}+\varepsilon Z_{3} & \varepsilon Z_{5}^{T} \\
Z_{5} & Z_{2}+\varepsilon Z_{4}
\end{array}\right], \quad E(\varepsilon)=\left[\begin{array}{cc}
I & 0 \\
0 & \varepsilon I
\end{array}\right]
$$

\section{Main Results}

3.1. Passivity Analysis and $\varepsilon$-Bound Estimation. In this subsection, using a novel storage function depending on the singular perturbation parameter, a sufficient condition for the system to be passive is proposed. Furthermore, bisectional search algorithms are formulated to get the best estimate of the $\varepsilon$-bound and the optimal dissipation $\eta$, respectively. 
Theorem 4. Given $\eta$ and $\bar{\varepsilon}>0$, system (1) with $u(t)=0$ is passive with dissipation $\eta$ for all $\varepsilon \in(0, \bar{\varepsilon}]$, if there exist scalars $\mu_{1}>0, \mu_{2}>0$, and matrices $Z_{i}(i=1, \ldots, 5)$ with $Z_{i}=$ $Z_{i}^{T}(i=1,2,3,4)$ satisfying the following LMIs:

$$
\begin{aligned}
& Z_{1}>0 \text {, } \\
& {\left[\begin{array}{cc}
Z_{1}+\bar{\varepsilon} Z_{3} & \bar{\varepsilon} Z_{5}^{T} \\
\bar{\varepsilon} Z_{5} & \bar{\varepsilon} Z_{2}
\end{array}\right]>0,} \\
& {\left[\begin{array}{cc}
Z_{1}+\bar{\varepsilon} Z_{3} & \bar{\varepsilon} Z_{5}^{T} \\
\bar{\varepsilon} Z_{5} & \bar{\varepsilon} Z_{2}+\bar{\varepsilon}^{2} Z_{4}
\end{array}\right]>0,} \\
& {\left[\begin{array}{cccc}
\mu_{1} \alpha^{2} M^{T} M+\mu_{2} \beta^{2} N^{T} N+\Delta(0) & Z^{T}(0) B_{\omega}-C^{T} & Z^{T}(0) B_{f} & 0 \\
* & 2 \eta I-D_{\omega}^{T}-D_{\omega} & 0 & -D_{g} \\
* & * & -\mu_{1} I & 0 \\
* & * & * & -\mu_{2} I
\end{array}\right]} \\
& <0 \text {, }
\end{aligned}
$$

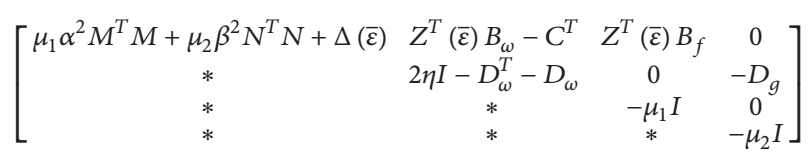$$
<0 \text {, }
$$

where $Z(\varepsilon)=\left[\begin{array}{cc}Z_{1}+\varepsilon Z_{3} & \varepsilon Z_{5}^{T} \\ Z_{5} & Z_{2}+\varepsilon Z_{4}\end{array}\right], \Delta(\varepsilon)=A^{T} Z(\varepsilon)+Z^{T}(\varepsilon) A$

Proof. Define a storage function as follows:

$$
V(x)=x^{T}(t) E(\varepsilon) Z(\varepsilon) x(t) .
$$

By Lemma 3, LMIs (11) imply that

$$
E(\varepsilon) Z(\varepsilon)=Z^{T}(\varepsilon) E(\varepsilon)>0, \quad \forall \varepsilon \in(0, \bar{\varepsilon}] .
$$
$(0, \bar{\varepsilon}]$.

Thus, $V(x)$ defined by (13) is positive definite for any $\varepsilon \epsilon$

Taking the derivative of $V(x)$ along the trajectories of system (1) with $u(t)=0$ and using constraints (2) and (3), for any scalars $\mu_{1}>0, \mu_{2}>0$, and $\varepsilon \in(0, \bar{\varepsilon}]$, we have

$$
\begin{aligned}
\left.\dot{V}(x)\right|_{(1)}= & \dot{x}^{T}(t) E(\varepsilon) Z(\varepsilon) x(t) \\
& +x^{T}(t) E(\varepsilon) Z(\varepsilon) \dot{x}(t) \\
= & \left(A x(t)+B_{f} f(t, x)+B_{\omega} \omega(t)\right)^{T} \\
& \times Z(\varepsilon) x(t) \\
& +x^{T}(t) Z^{T}(\varepsilon) \\
& \times\left(A x(t)+B_{f} f(t, x)+B_{\omega} \omega(t)\right) \\
= & x^{T}(t)\left(A^{T} Z(\varepsilon)+Z^{T}(\varepsilon) A\right) x(t) \\
& +2 x^{T}(t) Z^{T}(\varepsilon) B_{f} f(t, x) \\
& +2 x^{T}(t) Z^{T}(\varepsilon) B_{\omega} \omega(t) \\
\leq & x^{T}(t)\left(A^{T} Z(\varepsilon)+Z^{T}(\varepsilon) A\right) x(t) \\
& +2 x^{T}(t) Z^{T}(\varepsilon) B_{f} f(t, x) \\
& +2 x^{T}(t) Z^{T}(\varepsilon) B_{\omega} \omega(t) \\
& +\mu_{1}\left(\alpha^{2} x^{T}(t) M^{T} M x(t)-f^{T} f\right) \\
& +\mu_{2}\left(\beta^{2} x^{T}(t) N^{T} N x(t)-g^{T} g\right) .
\end{aligned}
$$

Therefore,

$$
\begin{aligned}
\left.\dot{V}(x)\right|_{(1)} & -2 \omega^{T}(t) z(t)+2 \eta \omega^{T}(t) \omega(t) \\
& =\left[\begin{array}{c}
x \\
\omega \\
f \\
g
\end{array}\right]^{T}\left[\begin{array}{cccc}
A^{T} Z(\varepsilon)+Z^{T}(\varepsilon) A+\Delta(\varepsilon) & Z^{T}(\varepsilon) B_{\omega}-C^{T} & Z^{T}(\varepsilon) B_{f} & 0 \\
* & 2 \eta I-D^{T}-D & 0 & -D_{g} \\
* & * & -\mu_{1} I & 0 \\
* & * & * & -\mu_{2} I
\end{array}\right]\left[\begin{array}{c}
x \\
\omega \\
f \\
g
\end{array}\right] .
\end{aligned}
$$

Taking into account (15) and (17), we have

$$
\left.\dot{V}(x)\right|_{(1)}-2 \omega^{T}(t) z(t)+2 \eta \omega^{T}(t) \omega(t) \leq 0 .
$$

Taking the integral on the two sides of (18) from 0 to $T$, we obtain

$$
\int_{0}^{T} \dot{V}(x)-2 \omega^{T}(t) z(t)+2 \eta \omega^{T}(t) \omega(t) \leq 0
$$


which implies

$$
\int_{0}^{T} 2 \omega^{T}(t) z(t)-2 \eta \omega^{T}(t) \omega(t) \geq V(x(T))-V(x(0)) .
$$

Therefore,

$$
\int_{0}^{T} \omega^{T}(t) z(t)-\eta \omega^{T}(t) \omega(t) d t \geq 0, \quad \forall T>0
$$

holds for all trajectories with zero initial condition $x(0)=0$. $(0, \bar{\varepsilon}]$.

Hence, system (1) is passive with dissipation $\eta$ for all $\varepsilon \in$

Remark 5. In [17], passivity of fuzzy SPSs was investigated based on decomposing the original system into fast and slow subsystems. Since the system decomposition requires the singular perturbation to be small enough, the proposed results are only sufficient conditions for the existence of an $\varepsilon$-bound for the SPSs to preserve passivity but cannot produce an estimate of the $\varepsilon$-bound. In this paper, the newly developed method implicitly employs the singular perturbation structure of the SPSs rather than depends on system decomposition, which provides convenience for estimating the value of the $\varepsilon$-bound.

Remark 6. In [19], a sufficient condition for system (1) to be passive with dissipation $\eta=0$ was proposed by using a storage function in the form of

$$
V(x)=\left[\begin{array}{ll}
x_{1}^{T} & x_{2}^{T}
\end{array}\right]\left[\begin{array}{cc}
Z_{1} & \varepsilon Z_{5}^{T} \\
\varepsilon Z_{5} & \varepsilon Z_{2}
\end{array}\right]\left[\begin{array}{l}
x_{1} \\
x_{2}
\end{array}\right]
$$

which is a special case of (13) with $Z_{3}=0$ and $Z_{4}=0$. Thus, Theorem 4 is more general and less conservative than the existing results in [19], which will be illustrated by the example in the next section.

The upper bound $\bar{\varepsilon}$ given by Theorem 4 is guessed. We now propose a bisectional search algorithm to get the best estimate of $\bar{\varepsilon}$.

Algorithm 7. Bisectional search algorithm optimizing $\bar{\varepsilon}$ for given dissipation $\eta$.

Step 1. Given positive scalars $\rho, \sigma, \gamma, \delta$, where $\rho, \delta$ are sufficiently small, $\gamma$ is sufficiently large, and $\rho<\sigma<\gamma$. Set $\underline{\tau}=\bar{\tau}=\sigma$.

Step 2. Check LMIs (11)-(12) with $\bar{\varepsilon}=\sigma$. If they are feasible, set $\underline{\tau}=\sigma$ and $\sigma:=2 \sigma$; otherwise, set $\bar{\tau}=\sigma$ and $\sigma:=0.5 \sigma$.

Step 3. If $\bar{\tau}=\rho$ or $\underline{\tau}>\gamma$, go to Step 7. Else if $\underline{\tau} \geq \bar{\tau}$, go to Step 2.

Step 4. Set $\varepsilon^{*}=0.5(\underline{\tau}+\bar{\tau})$.

Step 5. Check LMIs (11)-(12) with $\bar{\varepsilon}=\varepsilon^{*}$. If they are feasible, set $\underline{\tau}=\varepsilon^{*}$; otherwise, set $\bar{\tau}=\varepsilon^{*}$.

Step 6. Go to Step 4 if $|\underline{\tau}-\bar{\tau}|>\delta$; otherwise, go to Step 7 .
Step 7. If $\bar{\tau}<\rho$, the proposed method cannot give an answer. If $\underline{\tau}>\gamma$, the optimal upper bound $\bar{\varepsilon}$ is larger than $\gamma$. Otherwise, the optimal upper bound produced by the proposed method is the value of $\bar{\varepsilon}$. End.

Remark 8. In Algorithm 7, Step 1 presents the initial and terminal conditions. Steps 2-3 will show that the proposed method does not work or the optimal upper bound $\bar{\varepsilon}$ is larger than the given $\gamma$ or determine a search interval $[\underline{\tau}, \bar{\tau}]$ for Steps 4-6 such that LMIs (11)-(12) are feasible with $\bar{\varepsilon}=\underline{\tau}$ but not with $\bar{\varepsilon}=\bar{\tau}$. Steps $4-6$ are used to search the best estimate of the upper bound $\bar{\varepsilon}$ in $[\underline{\tau}, \bar{\tau}]$.

Similarly, we have the following algorithm to get the largest dissipation $\eta$ for given $\bar{\varepsilon}$.

Algorithm 9. Bisectional search algorithm optimizing $\eta$ for given $\bar{\varepsilon}$.

Step 1. Given positive scalars $\rho, \sigma, \gamma, \delta$, where $\rho, \delta$ are sufficiently small, $\gamma$ is sufficiently large, and $\rho<\sigma<\gamma$. Set $\underline{\tau}=\bar{\tau}=\sigma$.

Step 2. Check LMIs (11)-(12) with $\eta=\sigma$. If they are feasible, set $\underline{\tau}=\sigma$ and $\sigma:=2 \sigma$; otherwise, set $\bar{\tau}=\sigma$ and $\sigma:=0.5 \sigma$.

Step 3. If $\bar{\tau}=\rho$ or $\underline{\tau}>\gamma$, go to Step 7. Else if $\underline{\tau} \geq \overline{\bar{\tau}}$, go to Step 2.

Step 4. Set $\eta^{*}=0.5(\underline{\tau}+\bar{\tau})$.

Step 5. Check LMIs (11)-(12) with $\eta=\eta^{*}$. If they are feasible, set $\underline{\tau}=\eta^{*}$; otherwise, set $\bar{\tau}=\eta^{*}$.

Step 6. Go to Step 4 if $|\underline{\tau}-\bar{\tau}|>\delta$; otherwise, go to Step 7 .

Step 7. If $\bar{\tau}<\rho$, the proposed method cannot give an answer. If $\underline{\tau}>\gamma$, the optimal value of $\eta$ is larger than $\gamma$. Otherwise, the optimal value produced by the proposed method is the value of $\eta$. End.

Remark 10. Since the conditions proposed in Theorem 4 are sufficient but not necessary for the system to be passive, thus the best estimates of $\varepsilon$-bound and dissipation $\eta$ obtained by Algorithms 7 and 9 are less than or equal to their true values. Thus, the conservatism of the algorithms results from Theorem 4. Although Theorem 4 is less conservative than the existing results in [19], there may be some room to further reduce the conservatism, as will be considered in our future work.

3.2. Passive Controller Design. In this subsection, we will design a state feedback controller for system (1) to achieve a given $\varepsilon$-bound. The controller under consideration is in the form of

$$
u(t)=F(\varepsilon) x(t)
$$

where $F(\varepsilon) \in R^{l \times n}$ is the controller gain to be designed. Then, 
the closed-loop system is as follows:

$$
\begin{gathered}
E(\varepsilon) \dot{x}(t)=\left(A+B_{u} F(\varepsilon)\right) x(t)+B_{f} f(t, x)+B_{\omega} \omega(t), \\
z(t)=C x(t)+D_{g} g(t, x)+D_{\omega} \omega(t) .
\end{gathered}
$$

Theorem 11. Given $\eta$ and $\bar{\varepsilon}>0$, if there exist scalars $\mu_{1}>0$, $\mu_{2}>0, \bar{\mu}_{1}>0, \bar{\mu}_{2}>0$, and matrices $Z_{i}(i=1, \ldots, 5)$ with $Z_{i}=Z_{i}^{T}(i=1,2,3,4)$ and $K$ satisfying

$$
\begin{aligned}
& Z_{1}>0 \text {, } \\
& {\left[\begin{array}{cc}
Z_{1}+\bar{\varepsilon} Z_{3} & \bar{\varepsilon} Z_{5}^{T} \\
\bar{\varepsilon} Z_{5} & \bar{\varepsilon} Z_{2}
\end{array}\right]>0 \text {, }} \\
& {\left[\begin{array}{cc}
Z_{1}+\bar{\varepsilon} Z_{3} & \bar{\varepsilon} Z_{5}^{T} \\
\bar{\varepsilon} Z_{5} & \bar{\varepsilon} Z_{2}+\bar{\varepsilon}^{2} Z_{4}
\end{array}\right]>0 \text {, }} \\
& {\left[\begin{array}{cccccc}
\Theta(0) & B_{\omega}-Z^{T}(0) C^{T} & B_{f} & 0 & \alpha Z^{T}(0) M^{T} & \beta Z^{T}(0) N^{T} \\
* & 2 \eta I-D_{\omega}^{T}-D_{\omega} & 0 & -D_{g} & 0 & 0 \\
* & * & -\mu_{1} I & 0 & 0 & 0 \\
* & * & * & -\mu_{2} I & 0 & 0 \\
* & * & * & * & -\bar{\mu}_{1} I & 0 \\
* & * & * & * & * & -\bar{\mu}_{2} I
\end{array}\right]<0,} \\
& {\left[\begin{array}{cccccc}
\Theta(\bar{\varepsilon}) & B_{\omega}-Z^{T}(\bar{\varepsilon}) C^{T} & B_{f} & 0 & \alpha Z^{T}(\bar{\varepsilon}) M^{T} & \beta Z^{T}(\bar{\varepsilon}) N^{T} \\
* & 2 \eta I-D_{\omega}{ }^{T}-D_{\omega} & 0 & -D_{g} & 0 & 0 \\
* & * & -\mu_{1} I & 0 & 0 & 0 \\
* & * & * & -\mu_{2} I & 0 & 0 \\
* & * & * & * & -\bar{\mu}_{1} I & 0 \\
* & * & * & * & * & -\bar{\mu}_{2} I
\end{array}\right]<0 \text {, }} \\
& \mu_{1} \bar{\mu}_{1}=1, \quad \mu_{2} \bar{\mu}_{2}=1,
\end{aligned}
$$

where $Z(\varepsilon)=\left[\begin{array}{cc}Z_{1}+\varepsilon Z_{3} & \varepsilon Z_{5}^{T} \\ Z_{5} & Z_{2}+\varepsilon Z_{4}\end{array}\right], \Theta(\varepsilon)=A Z(\varepsilon)+Z^{T}(\varepsilon) A^{T}+$ $B K+K^{T} B^{T}$, then, the closed-loop system (24) is passive with dissipation $\eta$ for all $\varepsilon \in(0, \bar{\varepsilon}]$, and the gain matrix can be designed as $F(\varepsilon)=K Z^{-1}(\varepsilon)$.

Proof. By Lemma 3, LMIs (25) and (26) imply that

$$
E(\varepsilon) Z(\varepsilon)=Z^{T}(\varepsilon) E(\varepsilon)>0, \quad \forall \varepsilon \in(0, \bar{\varepsilon}],
$$

which shows that

$$
Z^{-T}(\varepsilon) E(\varepsilon)=E(\varepsilon) Z^{-1}(\varepsilon)>0, \quad \forall \varepsilon \in(0, \bar{\varepsilon}] .
$$

Define a storage function as follows:

$$
V(x)=x^{T}(t) Z^{-T}(\varepsilon) E(\varepsilon) x(t) .
$$

Thus, $V(x)$ defined by (32) is positive definite for any $\varepsilon \in$ $(0, \bar{\varepsilon}]$.

By Lemma 2, it follows from (27), (28), and (29) that, for any $\varepsilon \in(0, \bar{\varepsilon}]$,

$$
\left[\begin{array}{cccccc}
\Theta(\varepsilon) & B_{\omega}-Z^{T}(\varepsilon) C^{T} & B_{f} & 0 & \alpha Z^{T}(\varepsilon) M^{T} & \beta Z^{T}(\varepsilon) N^{T} \\
* & 2 \eta I-D_{\omega}{ }^{T}-D_{\omega} & 0 & -D_{g} & 0 & 0 \\
* & * & -\mu_{1} I & 0 & 0 & 0 \\
* & * & * & -\mu_{2} I & 0 & 0 \\
* & * & * & * & -\bar{\mu}_{1} I & 0 \\
* & * & * & * & * & -\bar{\mu}_{2} I
\end{array}\right]<0,
$$


which is equivalent to

$$
\left[\begin{array}{cccc}
\Theta(\varepsilon)+\mu_{1} \alpha^{2} Z^{T}(\varepsilon) M^{T} M Z(\varepsilon) & B_{\omega}-Z^{T}(\varepsilon) C^{T} & B_{f} & 0 \\
+\mu_{2} \beta^{2} Z^{T}(\varepsilon) N^{T} N Z(\varepsilon) & 2 \eta I-D_{\omega}{ }^{T}-D_{\omega} & 0 & -D_{g} \\
* & * & -\mu_{1} I & 0 \\
* & * & * & -\mu_{2} I
\end{array}\right]<0 .
$$

Pre- and postmultiplying (34) by $\operatorname{diag}\left\{Z^{-T}, I, I, I\right\}$ and its transpose gives

$$
\left[\begin{array}{cccc}
\bar{\Theta}(\varepsilon)+\mu_{1} \alpha^{2} M^{T} M & Z^{-T}(\varepsilon) B_{\omega}-C^{T} & Z^{-T}(\varepsilon) B_{f} & 0 \\
+\mu_{2} \beta^{2} N^{T} N & 2 \eta I-D_{\omega}{ }^{T}-D_{\omega} & 0 & -D_{g} \\
* & * & -\mu_{1} I & 0 \\
* & * & * & -\mu_{2} I
\end{array}\right]<0, \quad \varepsilon \in(0, \bar{\varepsilon}]
$$

where $\bar{\Theta}(\varepsilon)=Z^{-T}(\varepsilon)(A+B F(\varepsilon))+(A+B F(\varepsilon))^{T} Z^{-1}(\varepsilon), F(\varepsilon)=$ $K Z^{-1}(\varepsilon)$.

Taking the derivative of $V(x)$ along the trajectories of system (24) and using constraints (2) and (3), for any scalars $\mu_{1}>0, \mu_{2}>0$, and $\varepsilon \in(0, \bar{\varepsilon}]$, we have

$$
\begin{aligned}
\left.\dot{V}(x)\right|_{(22)}= & 2 x^{T}(t) Z^{-T}(\varepsilon) E(\varepsilon) \dot{x}(t) \\
= & 2 x^{T}(t) Z^{-T}(\varepsilon) \\
& \times\left((A+B F(\varepsilon)) x(t)+B_{f} f(t, x)+B_{\omega} \omega(t)\right) \\
= & x^{T}(t)\left(Z(\varepsilon)^{-T}(A+B F(\varepsilon))\right. \\
& \left.+(A+B F(\varepsilon))^{T} Z^{-1}(\varepsilon)\right) x(t) \\
& +2 x^{T}(t) Z^{-T}(\varepsilon) B_{f} f(t, x)
\end{aligned}
$$$$
\left.\dot{V}(x)\right|_{(22)}-2 \omega^{T}(t) z(t)+2 \eta \omega^{T}(t) \omega(t)
$$$$
=\left[\begin{array}{c}
x \\
\omega \\
f \\
g
\end{array}\right]^{T}\left[\begin{array}{cccc}
\bar{\Theta}(\varepsilon)+\mu_{1} \alpha^{2} M^{T} M & Z^{-T}(\varepsilon) B_{\omega}-C^{T} & Z^{-T}(\varepsilon) B_{f} & 0 \\
+\mu_{2} \beta^{2} N^{T} N & 2 \eta I-D_{\omega}{ }^{T}-D_{\omega} & 0 & -D_{g} \\
* & * & -\mu_{1} I & 0 \\
* & * & * & -\mu_{2} I
\end{array}\right]\left[\begin{array}{c}
x \\
\omega \\
f \\
g
\end{array}\right] .
$$

Therefore,

Taking into account (35) and (37), we have

$$
\int_{0}^{T} \dot{V}(x)-2 \omega^{T}(t) z(t)+2 \eta \omega^{T}(t) \omega(t) \leq 0
$$

$$
\left.\dot{V}(x)\right|_{(22)}-2 \omega^{T}(t) z(t)+2 \eta \omega^{T}(t) \omega(t) \leq 0 .
$$

Taking the integral on the two sides of (38) from 0 to $T$, which implies we obtain

$$
\int_{0}^{T} 2 \omega^{T}(t) z(t)-2 \eta \omega^{T}(t) \omega(t) \geq V(x(T))-V(x(0)) .
$$


Therefore,

$$
\int_{0}^{T} \omega^{T}(t) z(t)-\eta \omega^{T}(t) \omega(t) d t \geq 0, \quad \forall T>0
$$

holds for all trajectories with zero initial condition $x(0)=0$. $(0, \bar{\varepsilon}]$.

Hence, system (24) is passive with dissipation $\eta$ for all $\varepsilon \in$

Remark 12. It follows from LMIs (25) that $Z_{1}$ and $Z_{2}$ are nonsingular matrices, which guarantees that $Z(0)$ is nonsingular. Then, $F=K Z^{-1}(\varepsilon)$ is well defined for all $\varepsilon \in(0, \bar{\varepsilon}]$. Furthermore, it can be seen that the controller reduces to an $\varepsilon$-independent one, that is, $u=F x=K\left[\begin{array}{cc}Z_{1} & 0 \\ Z_{5} & Z_{2}\end{array}\right]^{-1} x$, when $\varepsilon$ is sufficiently small.

Using Theorem 4, we have the following corollary to design an $\varepsilon$-independent controller.

Corollary 13. Given $\eta$ and $\bar{\varepsilon}>0$, if there exist scalars $\mu_{1}>0$, $\mu_{2}>0, \bar{\mu}_{1}>0, \bar{\mu}_{2}>0$, and matrices $Z_{i}(i=1,2,5)$ with $Z_{i}=Z_{i}^{T}>0(i=1,2)$ and $K$ satisfying (27) and (29), then the closed-loop system (24) is passive with dissipation $\eta$ for all $\varepsilon \in$ $(0, \bar{\varepsilon}]$, and the gain matrix can be designed as $F=K\left[\begin{array}{ll}Z_{1} & 0 \\ Z_{5} & Z_{2}\end{array}\right]^{-1}$.

Remark 14. It is noted that the condition presented in Theorem 11 is not a convex set due to the equality constraints in (29). Several approaches have been proposed to solve such nonconvex feasibility problems, among which the cone complementarity linearization (CCL) method [22] is most commonly used. For computational simplicity, one can set $\mu_{1}=\bar{\mu}_{1}=\mu_{2}=\bar{\mu}_{2}=1$; then, the LMI conditions in Theorem 11 are easy to be solved by LMI toolbox. However, this may lead to conservatism.

\section{Example}

In this section, the RLC circuit shown in Figure 1 will be used to demonstrate the utility of the proposed method and show the advantages over the existing results.

In Figure $1, R, L$, and $C$ denote resistor, inductance, and capacitor, respectively. $I$ is the current, $V$ is voltage of the capacitor, $u$ is the input voltage, and $R_{\delta}$ is a nonlinear resistor with the voltage-current characteristics $V\left(R_{\delta}\right)=I+f(I)$, where $f(I)$ satisfies $\|f(I)\| \leq 0.5\|I\|$.

Let $L=1 H, R=10 \Omega$, and $C$ be a small positive scalar with $C=\varepsilon, x_{1}=I$, and $x_{2}=V$. Then, the circuit shown in Figure 1 can be described by the following singularly perturbed system

$$
\begin{gathered}
\dot{x}_{1}=-x_{1}-f\left(x_{1}\right)-x_{2}, \\
\varepsilon \dot{x}_{2}=x_{1}+0.1 x_{2}-0.1 \omega-0.1 u, \\
z=x_{1}+2 \omega,
\end{gathered}
$$

where $\omega(t) \in L_{2}^{1}[0, \infty)$ denotes the external disturbance.

We first show that using Theorem 4 , we can obtain a less conservative estimate of $\varepsilon$-bound than the existing methods in [19]. To do this, we will study the passivity of system (42)

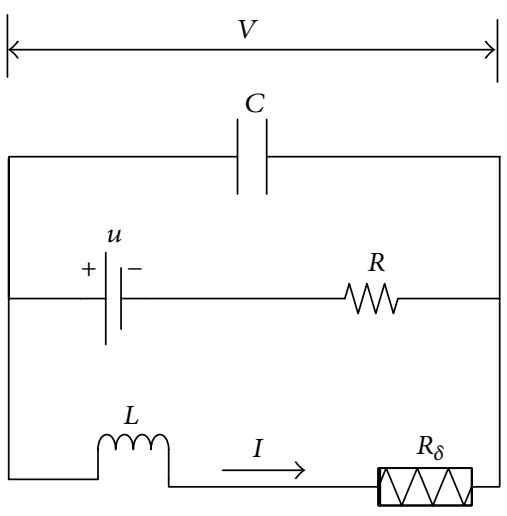

FIgure 1: An RLC circuit.

TABLE 1: Maximal $\bar{\varepsilon}$ for given $\eta$.

\begin{tabular}{lc}
\hline Given $\eta$ & Maximal $\bar{\varepsilon}$ \\
\hline 1.2 & 0.5849 \\
1.3 & 0.5571 \\
1.4 & 0.5198 \\
1.5 & 0.4666 \\
1.6 & 0.3817 \\
\hline
\end{tabular}

with $u=5 x_{1}+6 x_{2}$, which can be rewritten in the form of (1) with

$$
\begin{gathered}
E(\varepsilon)=\left[\begin{array}{ll}
1 & 0 \\
0 & \varepsilon
\end{array}\right], \quad A=\left[\begin{array}{cc}
-1 & -1 \\
0.5 & -0.5
\end{array}\right], \\
B_{u}=\left[\begin{array}{l}
0 \\
0
\end{array}\right], \quad B_{f}=\left[\begin{array}{c}
-1 \\
0
\end{array}\right], \quad B_{\omega}=\left[\begin{array}{c}
0 \\
-0.1
\end{array}\right], \\
C=\left[\begin{array}{ll}
1 & 0
\end{array}\right], \quad D_{g}=0, \quad D_{\omega}=2 .
\end{gathered}
$$

It is easy to verify that the nonlinearity $f\left(x_{1}\right)$ satisfies (2) with $\alpha=0.5$ and $M=\left[\begin{array}{ll}1 & 0\end{array}\right]$.

By the existing method of [19], the system is passive with dissipation $\eta=0$ for any $\varepsilon \in(0,0.6335]$.

Setting $\eta=0$ and using Algorithm 7, we have $\bar{\varepsilon}=0.7015$ and the corresponding solution to LMIs (11)-(12) is as follows:

$$
\begin{gathered}
Z_{1}=10.0359, \quad Z_{2}=25.4846, \quad Z_{3}=5.5166, \\
Z_{4}=8.0593, \quad Z_{5}=-17.4796, \\
\mu_{1}=7.3139, \quad \mu_{2}=5.4073 \times 10^{7} .
\end{gathered}
$$

It can be seen that Algorithm 7 leads to a tighter $\varepsilon$-bound than the existing method in [19].

By Algorithm 7, the best estimate of the $\varepsilon$-bound can be obtained for different values of dissipation $\eta$. By Algorithm 9, the best estimate of dissipation $\eta$ can be obtained for different value of $\bar{\varepsilon}$. The results are summarized in Tables 1 and 2, respectively, which shows that there is a competition between the dissipation $\eta$ and the upper bound $\bar{\varepsilon}$. 
TABLE 2: Maximal $\eta$ for given $\bar{\varepsilon}$.

\begin{tabular}{lc}
\hline Given $\bar{\varepsilon}$ & Maximal $\eta$ \\
\hline 0.2 & 1.6666 \\
0.3 & 1.6530 \\
0.4 & 1.5803 \\
0.5 & 1.4419 \\
0.6 & 1.1328 \\
\hline
\end{tabular}

Next, we demonstrate the utility of Theorem 11. System (42) can be rewritten in the form of (1) with

$$
\begin{gathered}
E(\varepsilon)=\left[\begin{array}{ll}
1 & 0 \\
0 & \varepsilon
\end{array}\right], \quad A=\left[\begin{array}{cc}
-1 & -1 \\
0.5 & -0.5
\end{array}\right], \\
B_{u}=\left[\begin{array}{c}
0 \\
-0.1
\end{array}\right], \quad B_{f}=\left[\begin{array}{c}
-1 \\
0
\end{array}\right], \quad B_{\omega}=\left[\begin{array}{c}
0 \\
-0.1
\end{array}\right], \\
C=\left[\begin{array}{ll}
1 & 0
\end{array}\right], \quad D_{g}=0, \quad D_{\omega}=2 .
\end{gathered}
$$

Letting $\bar{\varepsilon}=0.8, \eta=1.8$, and solving the LMI conditions in Theorem 11 with $\mu_{1}=\bar{\mu}_{1}=\mu_{2}=\bar{\mu}_{2}=1$, we have

$$
\begin{gathered}
Z_{1}=0.7339, \quad Z_{2}=10.5514, \quad Z_{3}=-0.2041 \\
Z_{4}=-1.5881, \quad Z_{5}=1.2496 \\
K=[85.5984-47.7435]
\end{gathered}
$$

Then, by Theorem 11, the controller (23) with

$$
\begin{aligned}
& F(\varepsilon)=\left[\begin{array}{ll}
85.5984 & -47.7435
\end{array}\right] \\
& \times\left[\begin{array}{cc}
0.7339-0.2041 \varepsilon & 1.2496 \varepsilon \\
1.2496 & 10.5514-1.5881 \varepsilon
\end{array}\right]^{-1}
\end{aligned}
$$

renders the closed-loop system passive with dissipation $\eta=$ 1.8 for all $\varepsilon \in(0,0.8]$.

\section{Conclusion}

In this paper, the problems of passive control and $\varepsilon$-bound estimation were investigated for singularly perturbed systems subject to nonlinear uncertainties. A storage function which gives full consideration of the singular perturbation structure was constructed, by which an $\varepsilon$-independent LMI-based passivity analysis method was proposed. And a bisectional search algorithm was formulated to get the best estimate of the $\varepsilon$-bound. The proposed method was shown to be less conservative than the existing results. Furthermore, a controller design method was proposed to achieve a predefined $\varepsilon$-bound. The presented RLC circuit has demonstrated the utility of the proposed results and shown the advantages over the existing results. In our future work, we will try to propose less conservative results and generalize the proposed results of this paper to more general nonlinear singularly perturbed systems.

\section{Acknowledgment}

This work was supported by the Fundamental Research Funds for the Central Universities (JC136662).

\section{References}

[1] P. V. Kokotović, H. K. Khalil, and J. O’Reilly, Singular Perturbation Methods in Control: Analysis and Design, Academic Press, New York, NY, USA, 1986.

[2] W.-H. Chen, S.-T. Yang, X. Lu, and Y. Shen, "Exponential stability and exponential stabilization of singularly perturbed stochastic systems with time-varying delay," International Journal of Robust and Nonlinear Control, vol. 20, no. 18, pp. 20212044, 2010.

[3] C. Yang, Q. Zhang, and L. Zhou, Stability Analysis and Design for Nonlinear Singular Systems, Springer, Heidelberg, Germany, 2012.

[4] F. Sun, L. Zhou, Q. Zhang, and Y. Shen, "Stability bound analysis and synthesis for singularly perturbed systems with time-varying delay," Mathematical Problems in Engineering, vol. 2013, Article ID 517258, 8 pages, 2013.

[5] L. Cao and H. M. Schwartz, "Complementary results on the stability bounds of singularly perturbed systems," IEEE Transactions on Automatic Control, vol. 49, no. 11, pp. 2017-2021, 2004.

[6] G. H. Yang and J. Dong, "Control synthesis of singularly perturbed fuzzy systems," IEEE Transactions on Fuzzy Systems, vol. 16, no. 3, pp. 615-629, 2008.

[7] C. Yang and Q. Zhang, "Multiobjective control for T-S fuzzy singularly perturbed systems," IEEE Transactions on Fuzzy Systems, vol. 17, no. 1, pp. 104-115, 2009.

[8] J. C. Willems, "Dissipative dynamical systems. I. General theory," Archive for Rational Mechanics and Analysis, vol. 45, pp. 321-351, 1972.

[9] J. C. Willems, "Dissipative dynamical systems. II. Linear systems with quadratic supply rates," Archive for Rational Mechanics and Analysis, vol. 45, pp. 352-393, 1972.

[10] H. K. Khalil, Nonlinear Systems, Prentice Hall, Upper Saddle River, NJ, USA, 3rd edition, 2002.

[11] C. Li and X. Liao, "Passivity analysis of neural networks with time delay," IEEE Transactions on Circuits and Systems II, Express Briefs, vol. 52, no. 8, pp. 471-475, 2005.

[12] D. Q. Wei and X. S. Luo, "Passivity-based adaptive control of chaotic oscillations in power system," Chaos, Solitons and Fractals, vol. 31, no. 3, pp. 665-671, 2007.

[13] Q. Li, Q. Zhang, N. Yi, and Y. Yuan, "Robust passive control for uncertain time-delay singular systems," IEEE Transactions on Circuits and Systems. I. Regular Papers, vol. 56, no. 3, pp. 653663, 2009.

[14] J. Lian and Z. Feng, "Passivity analysis and synthesis for a class of discrete-time switched stochastic systems with time-varying delay," Asian Journal of Control, vol. 15, no. 2, pp. 501-511, 2013.

[15] J. Lian, P. Shi, and Z. Feng, "Passivity and passification for a class of uncertain switched stochastic time-delay systems," IEEE Transactions on Cybernetics, vol. 43, no. 1, pp. 3-13, 2013.

[16] Z. G. Wu, P. Shi, H. Su, and J. Chu, "Passivity analysis for discrete-time stochastic Markovian jump neural networks with mixed time delays," IEEE Transactions on Neural Networks, vol. 22, no. 10, pp. 1566-1575, 2011.

[17] G. Calcev, R. Goret, and V. Wertz, "Passivity and fuzzy control of singularly perturbed systems," in Proceedings of the 38th IEEE 
Conference on Decision and Control, pp. 4364-4365, Phoenix, Aariz, USA, December 1999.

[18] Y. Wang, Q. Wang, P. Zhou, and D. Duan, "Delay-dependent passivity and passification for uncertain singularly perturbed Markovian jump systems with time-varying delay," Circuits, Systems, and Signal Processing, vol. 31, no. 6, pp. 2179-2194, 2012.

[19] Y. Gao, G. Lu, and Z. Wang, "Passivity analysis of uncertain singularly perturbed systems," IEEE Transactions on Circuits and Systems II: Express Briefs, vol. 57, no. 6, pp. 486-490, 2010.

[20] Y. Gao, B. Sun, and G. Lu, "Passivity-based integral slidingmode control of uncertain singularly perturbed systems," IEEE Transactions on Circuits and Systems II: Express Briefs, vol. 58, no. 6, pp. 386-390, 2011.

[21] S. Boyd, L. El Ghaoui, E. Feron, and V. Balakrishnan, Linear Matrix Inequalities in System and Control Theory, vol. 15, SIAM, Philadelphia, Pa, USA, 1994.

[22] L. El Ghaoui, F. Oustry, and M. Ait Rami, "A cone complementarity linearization algorithm for static output-feedback and related problems," IEEE Transactions on Automatic Control, vol. 42, no. 8, pp. 1171-1176, 1997. 


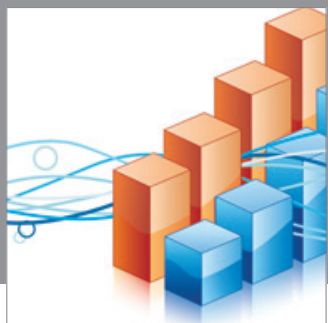

Advances in

Operations Research

mansans

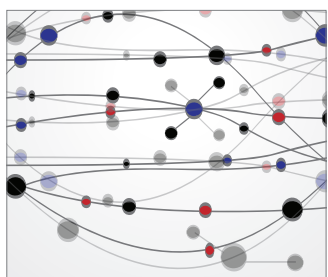

The Scientific World Journal
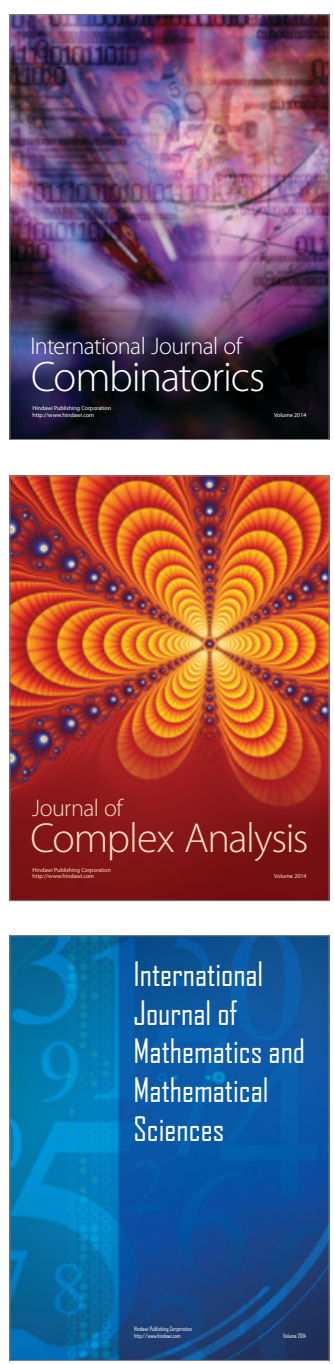
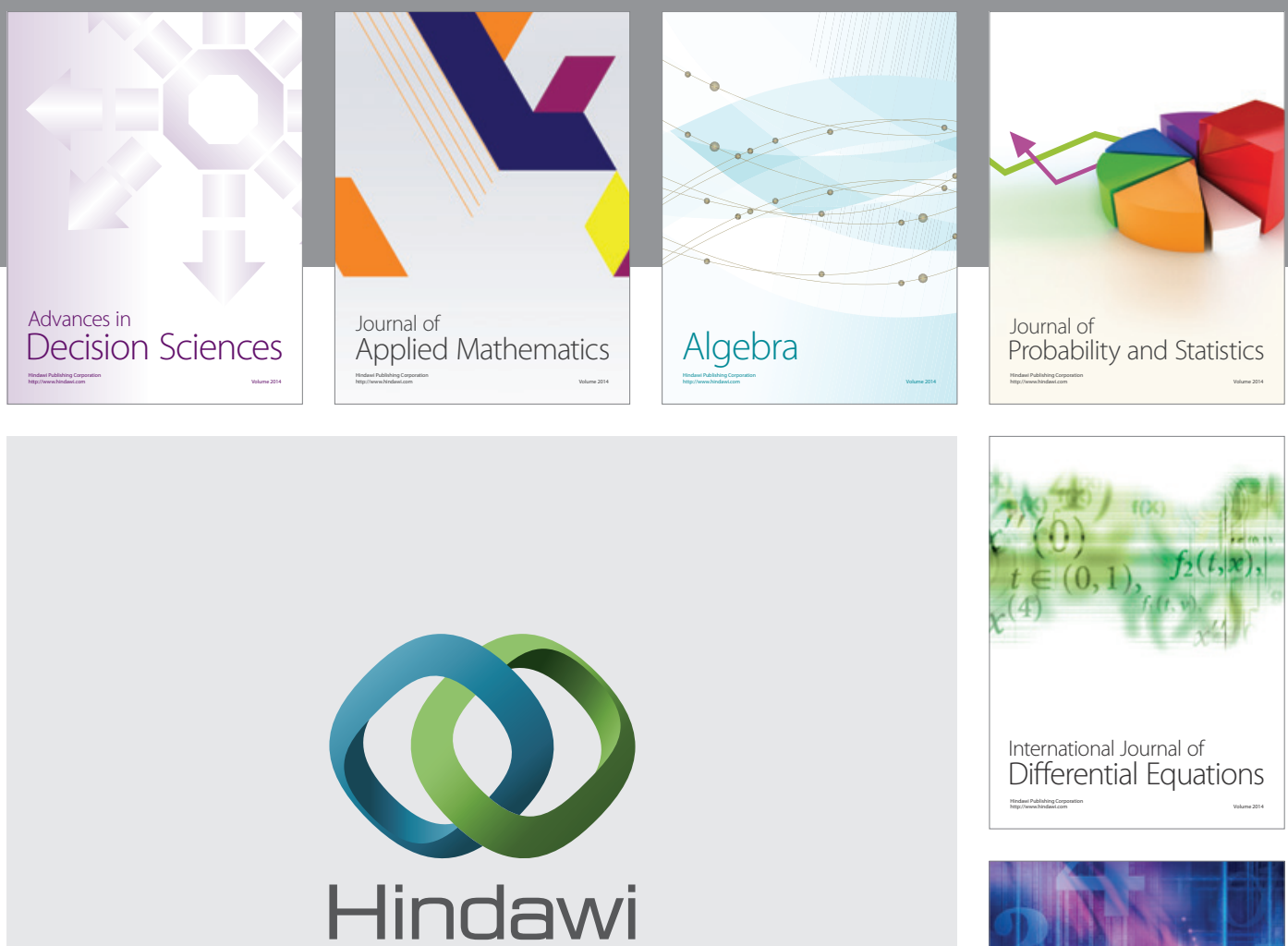

Submit your manuscripts at http://www.hindawi.com
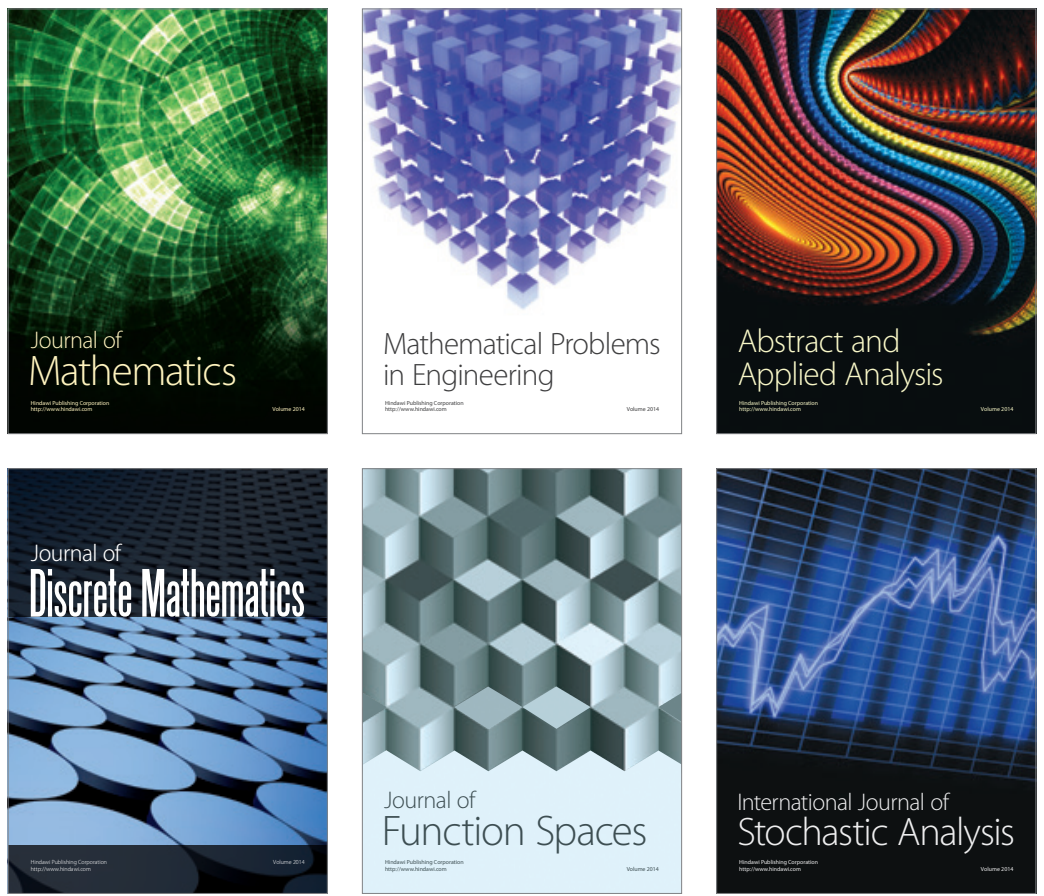

Journal of

Function Spaces

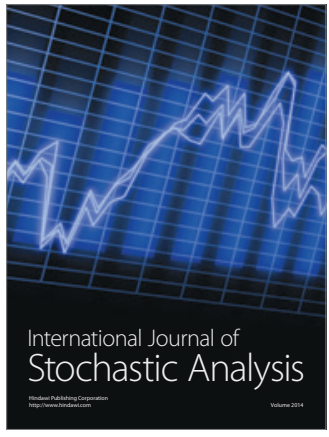

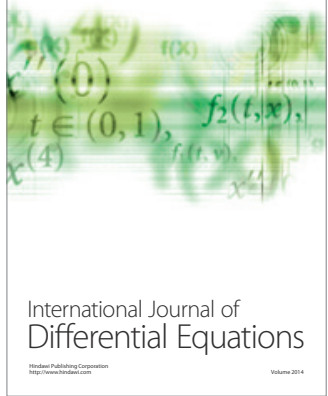
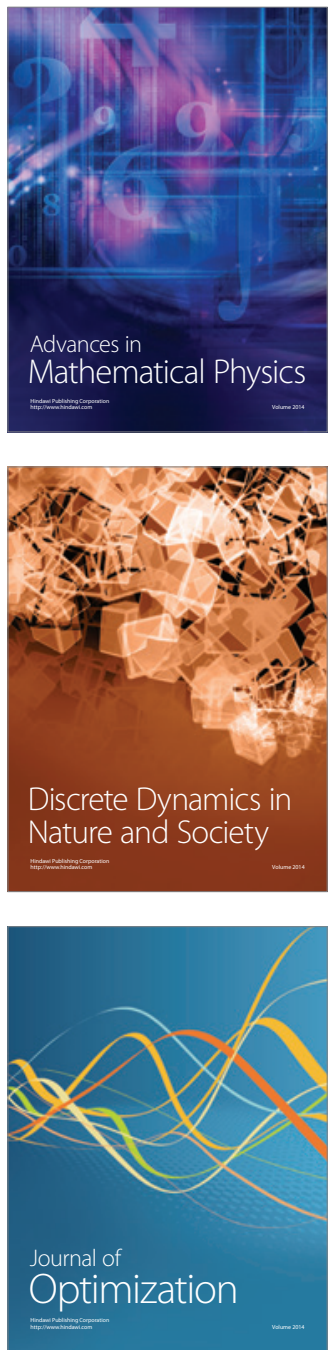